EGU21-2891

https://doi.org/10.5194/egusphere-egu21-2891

EGU General Assembly 2021

(c) Author(s) 2021. This work is distributed under

the Creative Commons Attribution 4.0 License.

\title{
The GCRF Living Deltas Hub: using water quality monitoring and lived experiences to achieve the UN Sustainable Development Goals
}

Lucy R. Roberts ${ }^{1}$, Heather L. Moorhouse ${ }^{2}$, Oanh Truong ${ }^{3}$, Phong Nguyen Thanh ${ }^{3}$, Suzanne McGowan ${ }^{1}$, Virginia N. Panizzo ${ }^{1}$, Phillip Barker ${ }^{2}$, Nga T. Do ${ }^{4,5}$, Feisal M.F. Rahman ${ }^{6}$, Jorge Salgado ${ }^{1}$, Tuhin Ghosh', Sourav Das ${ }^{7}$, Mashfiquas Salehin ${ }^{8}$, Ahmed Ishtiaque Amin Chowdhury ${ }^{8}$, Andrew C.G. Henderson ${ }^{9}$, and Andrew R.G. Large ${ }^{9}$

${ }^{1}$ School of Geography, University of Nottingham, Nottingham, NG7 2RD, UK

${ }^{2}$ Lancaster Environment Centre, Lancaster University, Lancaster, LA1 4YQ, UK

${ }^{3}$ Department of Management of Natural Resources and Environment, Hoa Sen university, Ho Chi Minh city, Vietnam

${ }^{4}$ Nuclear Training Center, Vietnam Atomic Energy Institute, 140 Nguyen Tuan, Thanh Xuan, Hanoi, Vietnam

${ }^{5}$ Electric Power University, 235 Hoang Quoc Viet, Cau Giay, Hanoi, Vietnam

${ }^{6}$ Department of Geography, Durham University, Lower Mountjoy, South Road, Durham, DH1 3LE, UK

${ }^{7}$ School of Oceanographic Sciences, Jadavpur University, Kolkata, 70032, India

${ }^{8}$ Institute of Water and Flood Management, Bangladesh University of Engineering and Technology, Dhaka, 1000,

Bangladesh

${ }^{9}$ School of Geography, Politics and Sociology, Newcastle University, Newcastle upon Tyne, NE1 7RU

The Living Deltas Hub is a UKRI GCRF-funded community investigating the environmental, societal, and natural-cultural heritage of three South and Southeast Asian mega-deltas; the GangesBrahmaputra-Meghna delta spanning India and Bangladesh, and the Mekong Delta and Red River Delta of Vietnam. Globally, deltas occupy only $1 \%$ of the total land area, but support the livelihoods of $\sim 500$ million citizens. As a consequence of growing human populations and intensified anthropogenic activity these deltas face multiple challenges, such as eustatic sea level rise, land subsidence, saline intrusion, unsustainable extraction of natural resources, habitat loss, pollution, and are currently on a trajectory towards collapse. The waterscape of the deltas place SDG 6 (clean water and sanitation) at the heart of sustainable development. Thus, the Hub aims to quantify and assess human impacts on the water quality of major river channels, canals, and ponds by establishing catchment-wide water quality monitoring supplemented by historical data, biomonitoring networks, community science projects (including water quality and participatory GIS) and local knowledge of water quality. This will result in improved understanding of the impacts of the multi-functionality of water sources in Asian mega-deltas from basic domestic use (bathing and drinking water) up to industrial scale aquaculture, and can lead to the success of SDG 6 (clean water), SDG 3 (good health and wellbeing), SDG 2 (zero hunger - here, through sustainable aquaculture), and SDG 14 (life below water). In addition, the combined methodology of water quality monitoring and understanding lived experiences can be used to identify the concerns of local communities, identify inequalities in the access to safe water (working towards SDG 10 
reduced inequalities) and understand the female experience (working towards SDG 5 gender inequality). Using a literature review of pond water quality and use in the delta regions and data from household surveys conducted in three regions of the Mekong Delta (Ben Tre, An Giang and Can Tho), we will use ponds as a case study to demonstrate how this approach can be used to improve understanding of community access to safe water. 